\title{
Flux-conserving diagrammatic formulation of optical spectroscopy of open quantum systems
}

\author{
Shaul Mukamel ${ }^{*, \dagger}$ and Michael Galperin*, \\ $\dagger$ Department of Chemistry, University of California Irvine, Irvine, CA 92697, USA \\ $\ddagger$ Department of Chemistry and Biochemistry, University of California San Diego, La Jolla, \\ CA 92093, USA \\ E-mail: smukamel@uci.edu; migalperin@ucsd.edu \\ Phone: +1 949824 7600; +1 8582460511
}




\begin{abstract}
We present a theoretical approach to optical spectroscopy of open nonequilibrium systems, which generalizes traditional nonlinear optical spectroscopy tools by imposing charge and energy conservation at all levels of approximation. Both molecular and radiation field degrees of freedom are treated quantum mechanically. The formulation is based on the nonequilibrium Green's function (NEGF) approach and a double sided Feynman diagrammatic representation of the photon flux is developed. Numerical simulations are presented for a model system. Our study bridges the theoretical approaches of quantum transport and optical spectroscopy and establishes a firm basis for applying traditional tools of nonlinear optical spectroscopy in molecular optoelectronics.
\end{abstract}

\title{
Introduction
}

Optical spectroscopy is a standard tool for probing and controlling electronic and vibrational structure and dynamics in molecular systems. For example, attosecond pulses make real-time observation of atomic scale electron dynamics possible, 1 localized surface plasmons allow to go beyond diffraction limit achieving single-molecule sensitivity, ${ }^{2}$ surface and tip enhanced Raman spectroscopy yields information on single molecule vibrational structure and excitations,,$\sqrt[3 / 4]{ }$ tetrahertz electromagnetic radiation provides access to rotational degrees of freedom of molecules ${ }^{5}$ and X-ray spectroscopy gives access to electronic transitions and nuclear dynamics. ${ }^{6 / 77}$ Recently, quantum effects of radiation have attracted attention as well. 8 [10

Advances in nanoscale fabrication techniques allow optical measurements in currentcarrying single-molecule junctions. In particular, bias-induced luminescence was used to observe vibrationally resolved features with sub-molecular precision, 11 14 visualize intermolecular dipole-dipole coupling, $\frac{15}{15}$ investigate energy transfer in molecular dimers, $\frac{16}{,}$ study selective triplet exciton formation in single molecule, $\frac{17}{17}$ and to access information on electronic quantum shot noise in the junction. ${ }^{18}$ Raman spectroscopy was utilized to resolve bias- 
dependent vibrational fingerprint of a molecule in a junction, 19 to observe time-dependent correlations between conductance and optical signal, $\stackrel{20}{\underline{2}}$ and to estimate extent of bias-induced vibrational and electronic heating in junctions. $\frac{21 \mid 22}{20}$ Optical read-out of the junction response to nanosecond voltage pulses was utilized to enable access to transient processes. $\stackrel{23}{\text { Perform- }}$ ing and interpreting optical experiments in open non-equilibrium molecular systems requires the combination of two research areas - optical spectroscopy and molecular electronics indicating the emergence of a new research direction coined molecular optoelectronics. 24

The theory of nonlinear optical spectroscopy of molecules is well established. $\frac{25}{33} \mathrm{~A}$ unifying framework for the interpretation of optical measurements in molecules was published in the book "Principles of Nonlinear Optical Spectroscopy", $\underline{34}$ the very classification of ultrafast optical processes is based on double-sided Feynman diagrams first introduced in the book. These represent a bare perturbation theory expansion of the molecular density matrix in light-matter interaction. Caution should be exercised with the approach in open systems. When the radiaton field is treated classically the bare perturbation expansion holds for closed and open systems alike, and the double-sided Feynman diagrams are constructed in the usual way. The only restriction is the necessity to avoid quantum regression statement, $\stackrel{35}{\text { when }}$ evaluating multi-time correlation functions of electronic operators. ${ }^{36}$ However, the treatment of quantum radiation fields is more involved ${ }^{37}$ Mutual influence of two quantum subsystems (e.g., radiation field and electronic degrees of freedom) leads to restrictions on building per-

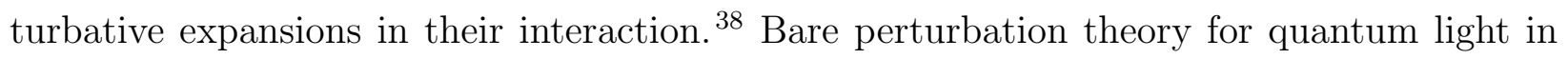
open systems does not conserve charge and energy $\underline{\sqrt[39]{42}}$ and may even lead to qualitative failures due to lack of account for back action from one system on the other in the bare $\operatorname{expansion} . \underline{43}$

Here, we develop a Green's function approach whereby charge and energy conservation are built in. That is, total charge and total energy in the whole system do not change during the evolution. A double sided Feynman diagrammatic expansion of the Green's functions that can describe the response of open systems to quantum fields in terms of pathways is 
developed.

The structure of the paper is as follows. After introducing a model of open system subjected to quantum radiation, we consider consider diagrammatic expansion in light-matter interaction in and discuss possible generalization of double-sided Feynman diagrams. Theoretical discussion is followed by illustrative numerical simulations. We conclude with short summary and directions for future research.

\section{Theoretical Methods}

\section{Model}

We consider a junction consisting of molecule $M$ coupled to two metallic contacts $L$ and $R$ (each at its own equilibrium) and to external quantum radiation field modes. The system Hamiltonian is

$$
\begin{aligned}
\hat{H} & =\hat{H}_{0}+\hat{V} \\
\hat{H}_{0} & =\hat{H}_{M}+\hat{H}_{L}+\hat{H}_{R}+\hat{H}_{r a d} \\
\hat{V} & =\hat{V}_{M L}+\hat{V}_{M R}+\hat{V}_{M, r a d}
\end{aligned}
$$

where $\hat{H}_{0}$ represents uncoupled molecule $\left(\hat{H}_{M}\right)$, contacts $\left(\hat{H}_{L}\right.$ and $\left.\hat{H}_{R}\right)$, and radiation field $\left(\hat{H}_{\text {rad }}\right)$, while $\hat{V}$ gives the interaction between the sub-systems. The molecular Hamiltonian $\hat{H}_{M}$ is assumed to be quadratic in the fermi operators (neglecting electron correlations), the contacts are modeled as continua of free charge carriers, the radiation field is expanded in a 
set of modes.

$$
\begin{aligned}
\hat{H}_{M} & =\sum_{m_{1}, m_{2}} H_{m_{2} m_{2}}^{M} \hat{d}_{m_{1}}^{\dagger} \hat{d}_{m_{2}} \\
\hat{H}_{K} & =\sum_{k \in K} \varepsilon_{k} \hat{c}_{k}^{\dagger} \hat{c}_{k} \\
\hat{H}_{\text {rad }} & =\sum_{\alpha} \omega_{\alpha} \hat{a}_{\alpha}^{\dagger} \hat{a}_{\alpha} \\
\hat{V}_{M K} & =\sum_{m \in M} \sum_{k \in K}\left(V_{m k} \hat{d}_{m}^{\dagger} \hat{c}_{k}+H . c .\right) \\
\hat{V}_{M, r a d} & =\sum_{m_{1}, m_{2} \in M} \sum_{\alpha}\left(U_{\alpha, m_{1} m_{2}} \hat{a}_{\alpha}^{\dagger} \hat{D}_{m_{1} m_{2}}+H . c .\right)
\end{aligned}
$$

Here $\hat{d}_{m}^{\dagger}\left(\hat{d}_{m}\right)$ and $\hat{c}_{k}^{\dagger}\left(\hat{c}_{k}\right)$ create (annihilate) electron in the molecular orbital $m$ or orbital $k$ of the contacts, respectively. $\hat{D}_{m_{1} m_{2}} \equiv \hat{d}_{m_{1}}^{\dagger} \hat{d}_{m_{2}}$ is the molecular de-excitation operator. $\hat{a}_{\alpha}^{\dagger}$ $\left(\hat{a}_{\alpha}\right)$ creates (annihilates) a photon in mode $\alpha$ of the radiation field.

We shall develop systematic approximations for electron and photon fluxes defined as the rate of change of population in contacts and radiation field respectively that conserve the fluxes, i.e. the charge and energy of the entire system does not change during evolution.

$$
\begin{aligned}
I_{K}(t) & \equiv-\frac{d}{d t} \sum_{k \in K}\left\langle\hat{c}_{k}^{\dagger}(t) \hat{c}_{k}(t)\right\rangle \quad(K=L, R) \\
I_{p t}(t) & \equiv+\frac{d}{d t} \sum_{\alpha}\left\langle\hat{a}_{\alpha}^{\dagger}(t) \hat{a}_{\alpha}\right\rangle
\end{aligned}
$$

and corresponding energy fluxes defined as rate of change of energy

$$
\begin{aligned}
& J_{K}(t) \equiv-\frac{d}{d t} \sum_{k \in K} \varepsilon_{k}\left\langle\hat{c}_{k}^{\dagger}(t) \hat{c}_{k}(t)\right\rangle \quad(K=L, R) \\
& J_{p t}(t) \equiv+\frac{d}{d t} \sum_{\alpha} \omega_{\alpha}\left\langle\hat{a}_{\alpha}^{\dagger}(t) \hat{a}_{\alpha}\right\rangle
\end{aligned}
$$

We adopt the conventional notation in quantum transport whereby positive electron flux is the flux from bath (contact) into system (molecule), while in optical spectroscopy positive 
photon flux goes from system (molecule) into bath (radiation field modes).

\section{Expanding the fluxes in the light-matter interaction}

The perturbative expansion is developed around the zero-order Hamiltonian $\hat{H}_{0}$. Standard nonequilibrium Green's function theory (NEGF) aims at calculating the electron and photon Green's functions defined on the Keldysh contour in the Heisenberg picture

$$
\begin{gathered}
G_{m_{1} m_{2}}\left(\tau_{1}, \tau_{2}\right) \equiv-i\left\langle T_{c} \hat{d}_{m_{1}}\left(\tau_{1}\right) \hat{d}_{m_{2}}^{\dagger}\left(\tau_{2}\right)\right\rangle \\
F_{\alpha_{1} \alpha_{2}}\left(\tau_{1}, \tau_{2}\right) \equiv-i\left\langle T_{c} \hat{a}_{\alpha_{1}}\left(\tau_{1}\right) \hat{a}_{\alpha_{2}}^{\dagger}\left(\tau_{2}\right)\right\rangle
\end{gathered}
$$

These satisfy set of exact coupled Dyson equations

$$
\begin{gathered}
\sum_{m} \int_{c} d \tau\left[\delta\left(\tau_{1}, \tau\right)\left(i \delta_{m_{1}, m} \frac{\partial}{\partial \tau}-H_{m_{1} m}^{M}\right)-\sum_{K=L, R} \Sigma_{m_{1} m}^{K}\left(\tau_{1}, \tau\right)\right] G_{m m_{2}}\left(\tau, \tau_{2}\right) \\
=\delta_{m_{1}, m_{2}} \delta\left(\tau_{1}, \tau_{2}\right)+\sum_{m} \int_{c} d \tau \Sigma_{m_{1} m}^{p t}\left(\tau_{1}, \tau\right) G_{m m_{2}}\left(\tau, \tau_{2}\right) \\
\left(i \frac{\partial}{\partial \tau_{1}}-\omega_{\alpha_{1}}\right) F_{\alpha_{1} \alpha_{2}}\left(\tau_{1}, \tau_{2}\right)=\delta_{\alpha_{1}, \alpha_{2}} \delta\left(\tau_{1}, \tau_{2}\right)+\sum_{\alpha} \int_{c} d \tau \Pi_{\alpha_{1} \alpha}^{e l}\left(\tau_{1}, \tau\right) F_{\alpha \alpha_{2}}\left(\tau, \tau_{2}\right)
\end{gathered}
$$

Here $\Sigma^{K}(K=L, R), \Sigma^{p t}$ and $\Pi^{e l}$ are self-energies of electrons due to coupling to contact $K$, electrons due to coupling to radiation field modes, and photons due to coupling to the electronic subsystem.

The bilinear molecule-contacts coupling, eq 7, results in an exact expression for the selfenergy $\Sigma^{K}$

$$
\sum_{m_{1} m_{2}}^{K}\left(\tau_{1}, \tau_{2}\right)=\sum_{k \in K} V_{m_{1} k} g_{k}\left(\tau_{1}, \tau_{2}\right) V_{k m_{2}}
$$

where $g_{k}\left(\tau_{1}, \tau_{2}\right) \equiv-i\left\langle T_{c} \hat{c}_{k}\left(\tau_{1}\right) \hat{c}_{k}^{\dagger}\left(\tau_{2}\right)\right\rangle$ is Green's function for free electrons in state $k$ of contact $K$. Its projections are $g_{k}^{r}\left(t_{1}, t_{2}\right)=-i \theta\left(t_{1}-t_{2}\right) e^{-i \varepsilon_{k}\left(t_{1}-t_{2}\right)}, g_{k}^{<}\left(t_{1}, t_{2}\right)=i n_{k} e^{-i \varepsilon_{k}\left(t_{1}-t_{2}\right)}$, $g_{k}^{>}\left(t_{1}, t_{2}\right)=-i\left[1-n_{k}\right] e^{-i \varepsilon_{k}\left(t_{1}-t_{2}\right)}$. The self-energy projections in the frequency domain are 
$(K=L, R)$

$$
\begin{aligned}
& \Sigma_{m_{1} m_{2}}^{K r}(E)=\Lambda_{m_{1} m_{2}}^{K}(E)-\frac{i}{2} \Gamma_{m_{1} m_{2}}^{K}(E) \\
& \Sigma_{m_{1} m_{2}}^{K<}(E)=i \Gamma_{m_{1} m_{2}}^{K}(E) f_{K}(E) \\
& \Sigma_{m_{1} m_{2}}^{K>}(E)=-i \Gamma_{m_{1} m_{2}}^{K}(E)\left[1-f_{K}(E)\right]
\end{aligned}
$$

Here $r,<$ and $>$ superscripts indicate retarded, lesser and greater projections, $f_{K}(E)$ is the Fermi-Dirac thermal distribution in the contacts.

$$
\Gamma_{m_{1} m_{2}}^{K}(E) \equiv 2 \pi \sum_{k \in K} V_{m_{1} k} V_{k m_{2}} \delta\left(E-\varepsilon_{k}\right)
$$

is a dissipation matrix due to coupling to contact $K$, and $\Lambda^{K}$ is the Lamb shift related to to $\Gamma^{K}$ via the Kramers-Kronig relations.

$\Sigma^{p t}$ and $\Pi^{e l}$ must be calculated approximately. Within the NEGF self-energies are defined as functional derivatives of the Luttinger-Ward functional $\Phi^{45-47}$ (see, e.g., eq 3.12 in Ref. 47)

$$
\begin{aligned}
& \Sigma_{m_{1} m_{2}}^{p t}\left(\tau_{1}, \tau_{2}\right)=+\frac{\delta \Phi}{\delta G_{m_{2} m_{1}}\left(\tau_{2}, \tau_{1}\right)} \\
& \Pi_{\alpha_{1} \alpha_{2}}^{e l}\left(\tau_{1}, \tau_{2}\right)=-\frac{\delta \Phi}{\delta F_{\alpha_{2} \alpha_{1}}\left(\tau_{2}, \tau_{1}\right)}
\end{aligned}
$$

Diagrams for the Luttinger-Ward functional to fourth order in light-matter interaction $\hat{V}_{M, r a d}$, eq 8 , are shown in Figure 1 .

$$
\begin{aligned}
\Phi & =i \sum_{\{\alpha\}} \sum_{\{m\}} \int_{c} d \tau_{1} \int_{c} d \tau_{2} U_{m_{1} m_{2}, \alpha_{1}} F_{\alpha_{1} \alpha_{2}}\left(\tau_{1}, \tau_{2}\right) U_{\alpha_{2}, m_{3} m_{4}} G_{m_{1} m_{3}}\left(\tau_{1}, \tau_{2}\right) G_{m_{4} m_{2}}\left(\tau_{2}, \tau_{1}\right) \\
& -\sum_{\{\alpha\}} \sum_{\{m\}} \int_{c} d \tau_{1} \int_{c} d \tau_{2} \int_{c} d \tau_{3} \int_{c} d \tau_{4} U_{m_{1} m_{2}, \alpha_{1}} F_{\alpha_{1} \alpha_{3}}\left(\tau_{1}, \tau_{3}\right) U_{\alpha_{3}, m_{3} m_{4}} \\
& \times U_{m_{5} m_{6}, \alpha_{2}} F_{\alpha_{2} \alpha_{4}}\left(\tau_{2}, \tau_{4}\right) U_{\alpha_{4}, m_{7} m_{8}} G_{m_{1} m_{6}}\left(\tau_{1}, \tau_{2}\right) G_{m_{5} m_{3}}\left(\tau_{2}, \tau_{3}\right) G_{m_{4} m_{7}}\left(\tau_{3}, \tau_{4}\right) G_{m_{8} m_{2}}\left(\tau_{4}, \tau_{1}\right)
\end{aligned}
$$


Self-energies constructed in this way are known to preserve all conservation laws in each order ${ }^{399}$ Explicit expressions for the self-energies to fourth order in $\hat{V}_{M, \text { rad }}$ are given in the Supporting Information.
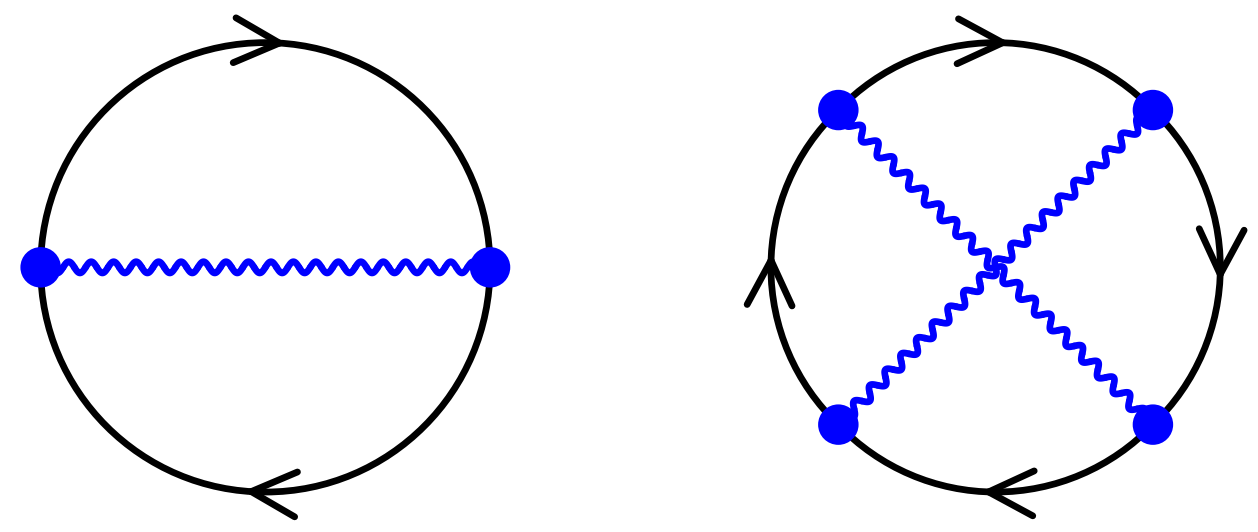

Figure 1: Diagrammatic perturbation theory within NEGF. Shown are the Luttinger-Ward generating functionals for second (left) and fourth (right) order expansion in light-matter interaction. Directed solid line (black) represents the electron Green function $G$, eq 13. Wavy line (blue) is the photon Green function $F$, eq 14 , both directions are implied here. Solid circles indicate vertices. Summation over all degrees of freedom and integration over contour variables is assumed at the vertices.

We note that the diagrammatic expansion is performed in the entire $V$, eq 3 , which includes both molecule-contacts and molecule-radiation field couplings. However, since the coupling to the contacts, eq 7, is quadratic, it is exactly resummed into the self-energy $\Sigma^{K}$, eq 17, while the molecule-radiation interaction can be accounted for through a perturbative expansion in the light-matter interaction.

Computing the Green's functions and self-energies is a bit different for time-dependent and steady-state applications. In the former case one has to solve time-dependent problem, which consists of setting initial conditions for the Green's functions. Because of causality self-energies required for a particular time step only depend on Green's functions at earlier times. So that starting from an initial condition one is able to propagate equations of motion step-by-step. Details of time propagation were discussed in, e.g., Ref. 48, Note that the initial condition may include either decoupled system and baths (contacts and radiation field) with sudden or adiabatic switching of the coupling, or steady-state junction (coupling 
to contacts switched at infinite past) subjected at time $t_{0}$ to laser pulse. Also propagation on two-dimensional time grid is extremely heavy numerically, so that approximate schemes reducing to a single time propagation were developed. 49

For steady-state, coupling to both contacts and radiation field are assumed to happen in the infinite past, and particular form of the switching (sudden or adiabatic) is not important because by the time steady-state was established, transients die out. In this case we Fourier transform the Dyson equations, Green's functions and self-energies to energy space. Dyson equations, eqs 15, 16, with self-energies, eqs S1-S2, have to be solved self-consistently until convergence starting from Green's function for, e.g., decoupled electronic and photon systems. In summary, such procedure consists of the following steps:

1. Obtain Green's functions for decoupled electrons and photons (e.g., solve problem for molecular junction in the absence of the field to get electron Green's function and assume free photon field - e.g., CW laser, for photon Green's function).

2. Use the Green's functions to evaluate the self-energies, eqs S1-S2.

3. Use the self-energies to calculate Green's functions by numerically solving the Dyson equations, eqs 15,16 .

4. Check convergence by, e.g., calculating populations of electronic levels and photon modes. If difference on two steps of the procedure is less than predefined tolerance, stop the calculation; otherwise return to step 2 .

Once the self-energies and Green's functions are known, one can calculate the fluxes, eqs 9 . 12. Within NEGF exact expressions for the fluxes are obtained by following the celebrated 
Jauho-Wingreen-Meir derivation $\underline{4450 / 51}$

$$
\begin{aligned}
& I_{K}(t)=2 \operatorname{Re} \int_{-\infty}^{t} d t^{\prime} \operatorname{Tr}\left[\Sigma^{K<}\left(t, t^{\prime}\right) G^{>}\left(t^{\prime}, t\right)-\Sigma^{K>}\left(t, t^{\prime}\right) G^{<}\left(t^{\prime}, t\right)\right] \\
& I_{p t}(t)=2 \operatorname{Re} \int_{-\infty}^{t} d t^{\prime} \operatorname{Tr}\left[F^{<}\left(t, t^{\prime}\right) \Pi^{e l>}\left(t^{\prime}, t\right)-F^{>}\left(t, t^{\prime}\right) \Pi^{e l<}\left(t^{\prime}, t\right)\right] \\
& J_{K}(t)=2 \operatorname{Im} \int_{-\infty}^{t} d t^{\prime} \operatorname{Tr}\left[\frac{\partial \Sigma^{K>}\left(t, t^{\prime}\right)}{\partial t} G^{<}\left(t^{\prime}, t\right)-\frac{\partial \Sigma^{K}<\left(t, t^{\prime}\right)}{\partial t} G^{>}\left(t^{\prime}, t\right)\right] \\
& J_{p t}(t)=2 \operatorname{Im} \int_{-\infty}^{t} d t^{\prime} \operatorname{Tr}\left[\frac{\partial F^{>}\left(t, t^{\prime}\right)}{\partial t} \Pi^{e l<}\left(t^{\prime}, t\right)-\frac{\partial\left[F^{<}\left(t, t^{\prime}\right)\right.}{\partial t} \Pi^{e l>}\left(t^{\prime}, t\right)\right] \\
&+2 \operatorname{Re} \int_{-\infty}^{t} d t^{\prime} \int_{-\infty}^{t^{\prime}} d t^{\prime \prime} \operatorname{Tr}\left[\Pi^{e l<}\left(t, t^{\prime \prime}\right) F^{>}\left(t^{\prime \prime}, t^{\prime}\right) \Pi^{e l>}\left(t^{\prime}, t\right)\right. \\
&\left.+\Pi^{e l>}\left(t, t^{\prime \prime}\right) F^{<}\left(t^{\prime \prime}, t^{\prime}\right) \Pi^{e l<}\left(t^{\prime}, t\right)\right]
\end{aligned}
$$

Here the trace is over molecular orbitals in eqs 25 and 27 and over radiation field modes in eqs 26 and 28. Note that the fluxes are coupled, because the self-energies entering their definitions are derived form the same Luttinger-Ward functional (see Figure 1). Thus, they should be treated on equal footing. This interdependence of fluxes results in charge and energy conservation (see below for a simple illustration). Note that in the usual NEGF approach the molecule-contacts coupling is switched on at the infinite past - thus minus infinity as lower limit in integrals in eqs 25,28. However, other switchings are possible.

\section{Results and Discussion}

\section{Double-sided Feynman diagrams for the Green's functions}

Below we present a double-sided Feynman diagram expansion of the fluxes, based on the diagrammatic expansion of the self-energies. It is important to stress the difference in language between Green's function (Hilbert space) and density matrix (Liouville space) formulations.

Original double-sided Feynman diagrams act in Liouville space. Corresponding construc- 


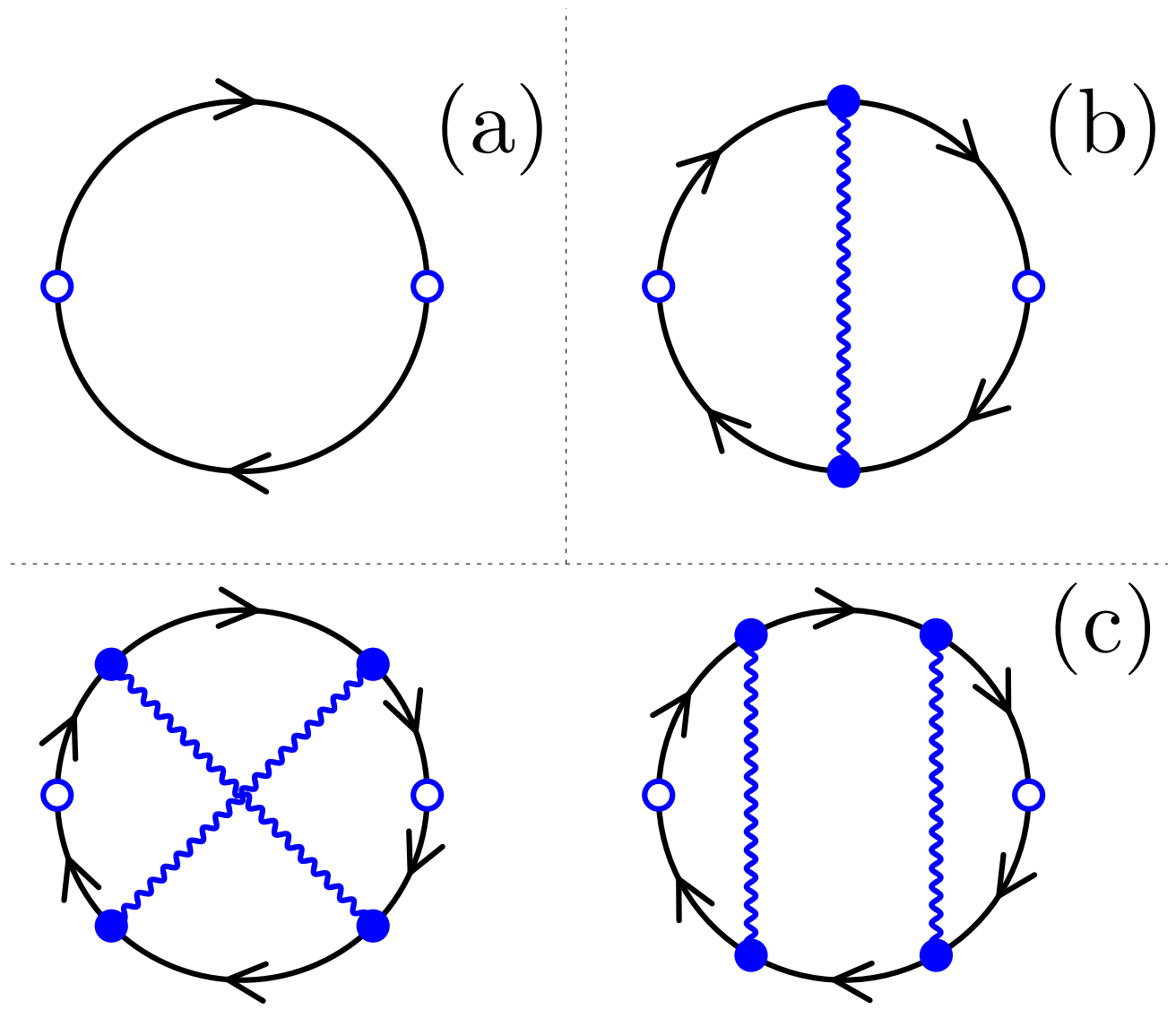

Figure 2: Diagrams contributing to photon self-energy due to coupling to electrons $\Pi^{e l}$. Shown are contributions of (a) second, (b) fourth, and (c) sixth orders. Directed solid line (black) represents the electron Green function $G$, eq 13. Wavy line (blue) is the photon Green function $F$, eq 14 , both directions are implied here. Open and solid circles indicate outer and inner vertices. Summation over all degrees of freedom and integration over contour variables is assumed for inner vertices. 
tion in the Hilbert space within Green's function technique is called projection, while term diagram is reserved for representation of irreducible contributions within perturbative expansion. Figure 2 shows second (a), fourth (b), and sixth (c) order Feynman diagrams contributing to photon self-energy due to coupling to electrons, $\Pi^{e l}$. Each diagram can be projected on the Keldysh contour resulting in a set of contributions, which in Liouville space language are denoted double-sided Feynman diagrams. An important point is that while in second and fourth order, where only one diagram contributes to the self-energy, difference in the languages is of secondary importance, one has to be careful with sixth order contribution, where two different diagrams (see Figure 20) representing different physical processes will have same set of time projections. Another difference to keep in mind is time ordering in the two approaches: while Green's function projections only account for ordering along the Keldysh contour, Liouville space formulation requires also ordering in physical time. Thus, one Hilbert space projection represents several Liouville space diagrams (see, e.g., Ref. 52 for more details).

We are now ready to introduce double-sided Feynman diagrams for the photon flux, eq26. Indeed, double-sided Feynman diagrams were originally introduced as contributions to the flux. ${ }^{34}$ In this expression we substitute photon self-energy with its explicit expression, eq S2, separating orders of contributions to the latter. Projections of contributions of different orders will yield analog of double-sided Feynman diagrams corresponding to optical processes at the order of the diagram. For example, second order double-sided Feynman diagram results from second order contribution to $\Pi^{e l}$ - first term in the right-hand-side of eq S2:

$$
\begin{aligned}
I_{p t}^{(2)}(t) & =2 \operatorname{Im} \int_{-\infty}^{t} d t^{\prime} \sum_{\alpha_{1}, \alpha_{2}} \sum_{\substack{n_{1}, n_{2} \in M \\
n_{3}, n_{4}}} U_{\alpha_{1}, n_{1} n_{2}} U_{n_{3} n_{4}, \alpha_{2}} \\
& \times\left(G_{n_{2} n_{4}}^{<}\left(t, t^{\prime}\right) G_{n_{3} n_{1}}^{>}\left(t^{\prime}, t\right) F_{\alpha_{2}, \alpha_{1}}^{>}\left(t^{\prime}, t\right)-G_{n_{2} n_{4}}^{>}\left(t, t^{\prime}\right) G_{n_{3} n_{1}}^{<}\left(t^{\prime}, t\right) F_{\alpha_{2}, \alpha_{1}}^{<}\left(t^{\prime}, t\right)\right)
\end{aligned}
$$

The corresponding double-sided Feynman diagrams are shown in Figure 3. Two additional diagrams (accounted for by Im ... in the expression above) are obtained by switching contour 

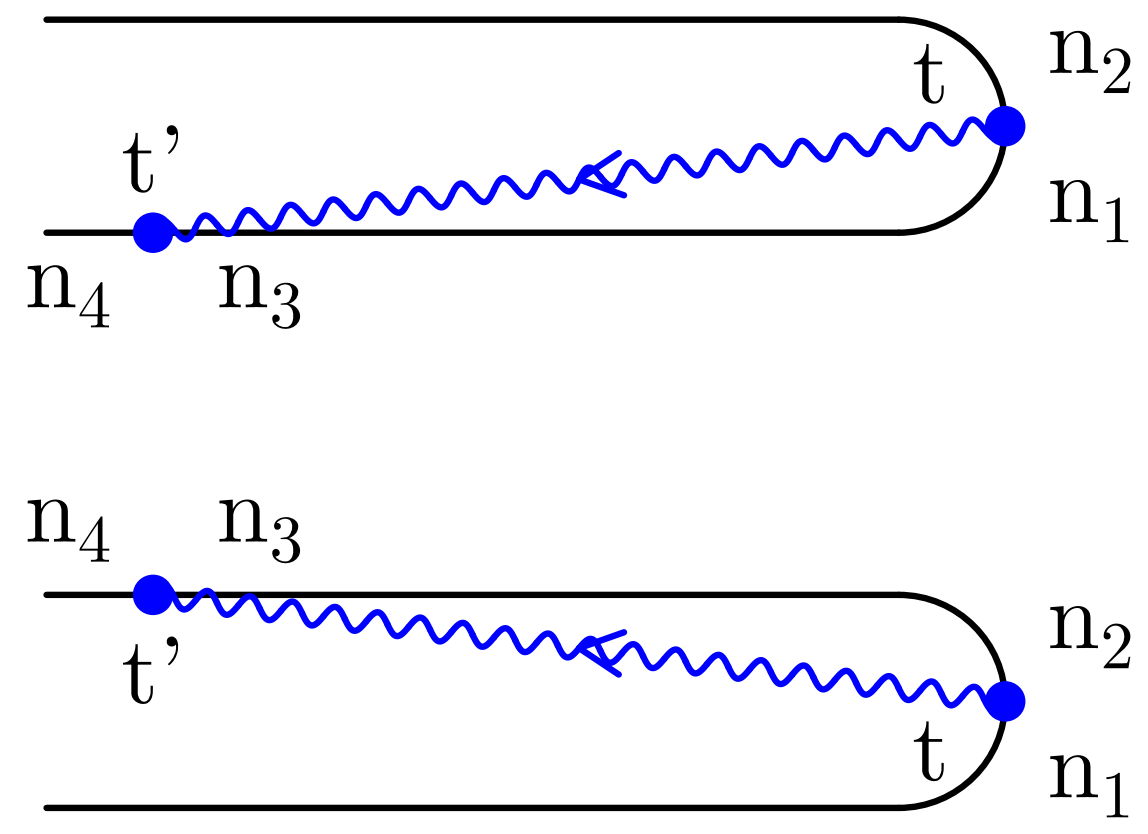

Figure 3: Double-sided Feynman diagrams for second order optical processes in the photon flux, eq 29. Wavy line (blue) is the photon Green function $F$, eq 14. Top (bottom) diagram corresponds to first (second) term in the right side of eq 29. Indices $n_{i}$ indicate molecular orbitals. 

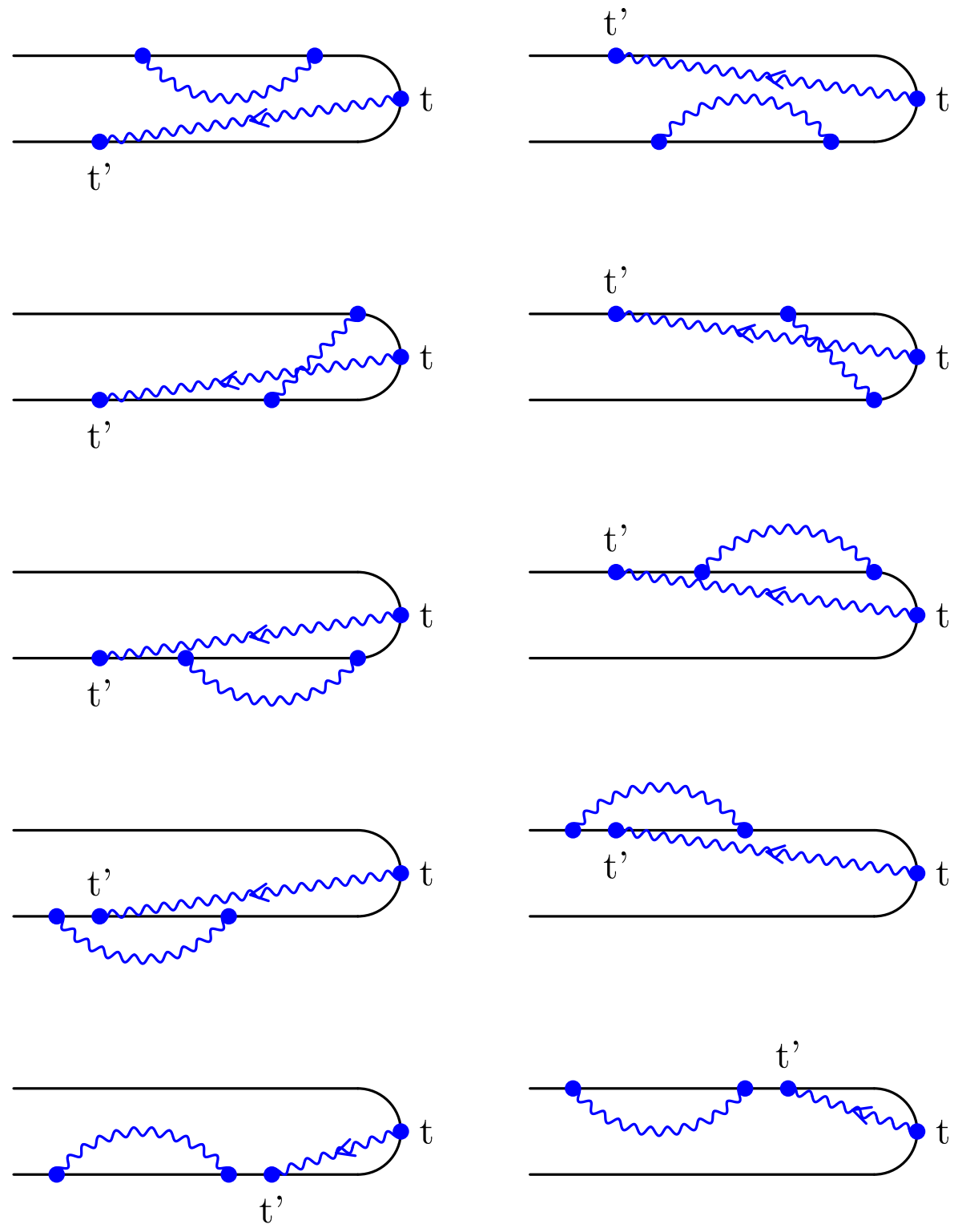

Figure 4: Double-sided Feynman diagrams for fourth order optical processes in the photon flux. Wavy line (blue) is the photon Green's function $F$, eq 14. Left (right) column corresponds to first (second) term in the right side of eq 26. Wavy line without arrow stands for $F\left(\tau_{3}, \tau_{4}\right)$ of eq $\mathrm{S} 2$. Both arrow directions are possible in this line. 
branches and flipping arrows in the photon Green's function.

Similarly, fourth order double sided Feynman diagrams are obtained by substituting fourth order contribution to self-energy $\Pi^{e l}$, second term in the right side of eq S2, into expression for photon flux, eq 10. Corresponding diagrams are shown in Figure 4. Note, only projections along the contour (Green's function Hilbert space projections) are shown.

We note that simulating double-sided Feynman diagrams following bare perturbation expansion is not feasible also due to the fact that such expansion takes into account also decoupled diagrams which should not contribute. Complicated subtraction of terms should be performed in such expansion as was discussed in Refs. $\stackrel{53154}{ }$ The problem does not appear in the present Green's function based approach.

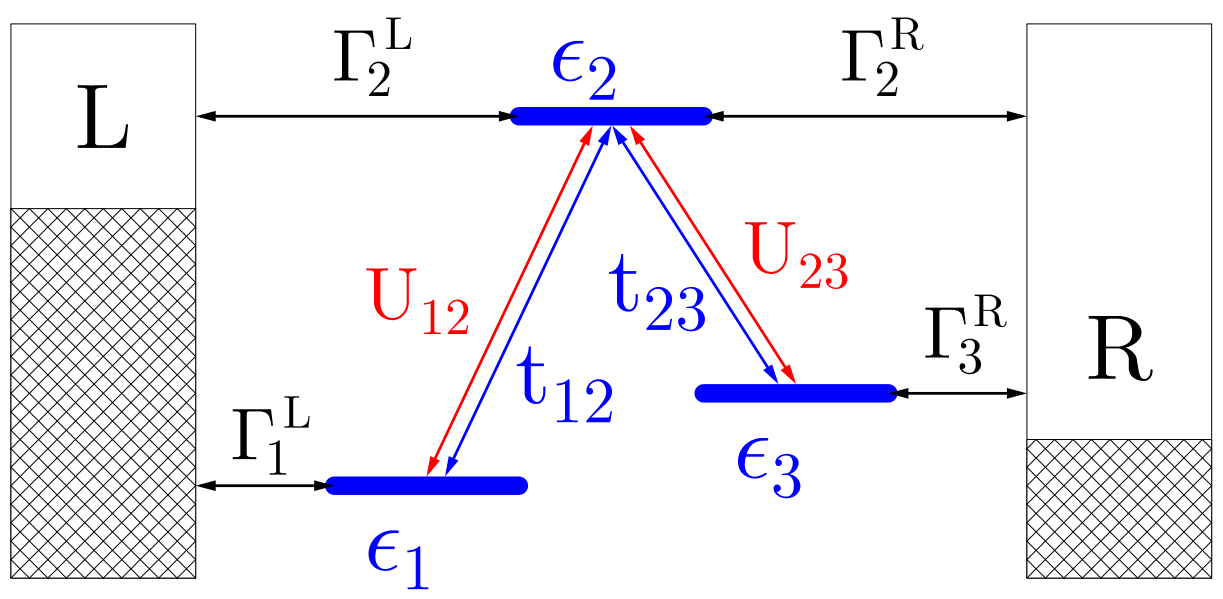

Figure 5: Donor (1) - bridge (2) - acceptor (3) junction model for photo-assisted electron transport.

\section{Numerical example}

The following simulations of particle and energy fluxes illustrate the conserving character of the double-sided Feynman diagram approach. We assume a three level model representing donor-bridge-acceptor (DBA) molecular structure with donor coupled to left and acceptor to right contacts. Bridge is assumed to be weakly coupled to both contacts (Figure 5). The donor and acceptor energies $\left(\varepsilon_{1}\right.$ and $\left.\varepsilon_{3}\right)$ are lower than the bridge energy $\left(\varepsilon_{2}\right)$. The system is 
subjected to external radiation which facilitates electron transfer from donor to bridge and from bridge to acceptor (see Figure 5). The Hamiltonian is

$$
\begin{aligned}
\hat{H}_{M} & =\sum_{m=1}^{3} \varepsilon_{m} \hat{d}_{m}^{\dagger} \hat{d}_{m}+\sum_{m=1}^{2}\left(t_{m, m+1} \hat{d}_{m}^{\dagger} \hat{d}_{m}+H . c .\right) \\
\hat{V}_{M L} & =\sum_{\ell i n L}\left(V_{1 \ell} \hat{d}_{1}^{\dagger} \hat{c}_{\ell}+V_{2 \ell} \hat{d}_{2}^{\dagger} \hat{c}_{\ell}+H . c .\right) \\
\hat{V}_{M R} & =\sum_{r \in R}\left(V_{3 r} \hat{d}_{3}^{\dagger} \hat{c}_{r}+V_{2 r} \hat{d}_{2}^{\dagger} \hat{c}_{r}+H . c .\right) \\
\hat{V}_{M, r a d} & =\sum_{\alpha}\left(U_{\alpha, 12} \hat{a}_{\alpha}^{\dagger} \hat{d}_{1}^{\dagger} \hat{d}_{2}+U_{\alpha, 32} \hat{a}_{\alpha}^{\dagger} \hat{d}_{3}^{\dagger} \hat{d}_{2}+H . c .\right)
\end{aligned}
$$

A similar model was used in Ref. 42, where non-conserving character of standard tools of nonlinear optical spectroscopy was illustrated. Here we demonstrate that the present expansion satisfies conservation laws.

We focus on steady-state and check the conservation of charge

$$
I_{L}=-I_{R}
$$

and energy

$$
J_{L}+J_{R}-J_{p t}=0
$$

Note that the minus sign in the energy balance is due to opposite convention about flux positivity for electron fluxes (positive is flux going into the system) and photons (positive is flux going out of the system). At steady state. all fluxes eqs 25.28, are time-independent. They can be expressed in terms of Fourier transforms of corresponding Green's functions 

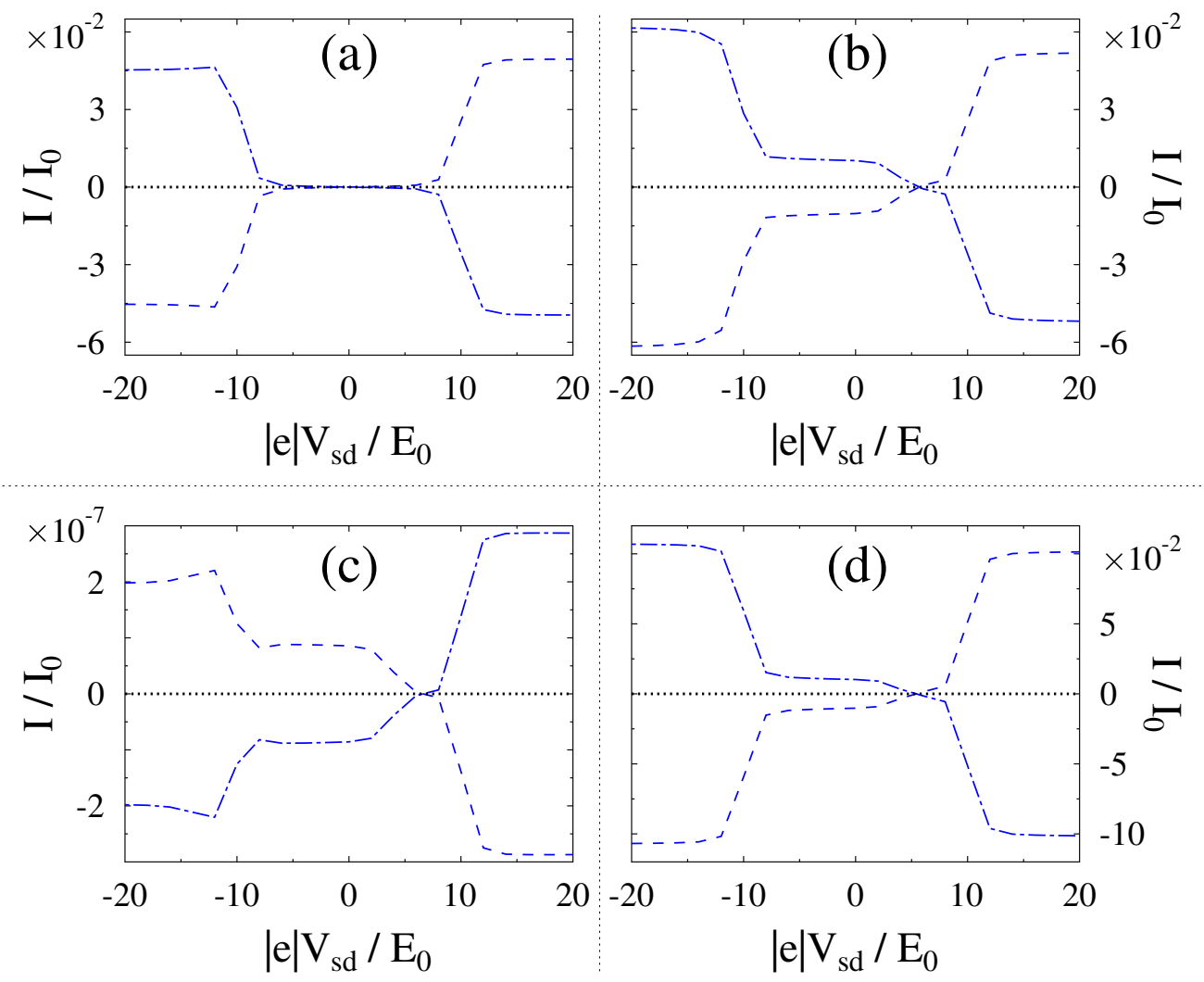

Figure 6: Charge conservation, eq 34, for the junction model of Figure 5. Shown are $I_{L}$ (dashed line, blue), $I_{R}$ (dash-dotted line, blue) and their sum (dotted line, black) for (a) zero, (b) second. and (c) fourth order contributions; (d) shows total fluxes, eq 36 . See text for parameters. 
and self-energies as $(K=L, R)$

$$
\begin{aligned}
I_{K} & =\int_{-\infty}^{+\infty} \frac{d E}{2 \pi} i_{K}(E) \\
I_{p t} & =\int_{-\infty}^{+\infty} \frac{d \omega}{2 \pi} i_{p t}(\omega) \\
J_{K} & =\int_{-\infty}^{+\infty} \frac{d E}{2 \pi} E i_{K}(E) \\
J_{p t} & =\int_{-\infty}^{+\infty} \frac{d \omega}{2 \pi} \omega i_{p t}(\omega)
\end{aligned}
$$

where

$$
\begin{aligned}
& i_{K}(E) \equiv \operatorname{Tr}\left[\Sigma_{K}^{<}(E) G^{>}(E)-\Sigma_{K}^{>}(E) G^{<}(E)\right] \\
& i_{p t}(\omega) \equiv \operatorname{Tr}\left[F^{<}(\omega) \Pi^{>}(\omega)-F^{>}(\omega) \Pi^{<}(\omega)\right]
\end{aligned}
$$

The radiation field is described as set of modes (oscillators). populated by CW laser characterized by its frequency $\omega_{0}$, intensity $N_{0}$, and bandwidth $\delta$, so that the population $N_{p t}(\omega)$ is

$$
N_{p t}(\omega)=N_{0} \frac{\delta^{2}}{\left(\omega-\omega_{0}\right)^{2}+\delta^{2}}
$$

Further details of the steady-state simulation can be found in Ref. 42 .

The simulation parameters are (all numbers are given in terms of arbitrary unit of energy $\left.E_{0}\right): k_{B} T=0.25, \varepsilon_{1}=-5, \varepsilon_{2}=5, \varepsilon_{3}=-2, t_{12}=t_{23}=0.1 . \quad \Gamma_{1}^{L}=\Gamma_{3}^{R}=1$ and $\Gamma_{2}^{L}=\Gamma_{2}^{R}=0.1$ are electron escape rates from donor, bridge and acceptor into left and right contacts. $\gamma_{0}=0.1$ is energy escape rate from the molecule into radiation field modes. The molecule is subjected to external laser radiation with frequency $\omega_{0}=7$ and width $\delta=0.1$. The laser frequency is chosen at resonance for the transition between bridge and acceptor. Fermi energy is taken as the origin, $E_{F}=0$, and bias is assumed to be applied symmetrically, $\mu_{L / R}=E_{F} \pm|e| V_{s d} / 2$. Simulations were performed on energy grid spanning region from -15 to +15 with step 0.01 . Self-consistent NEGF simulation was assumed to converge when 

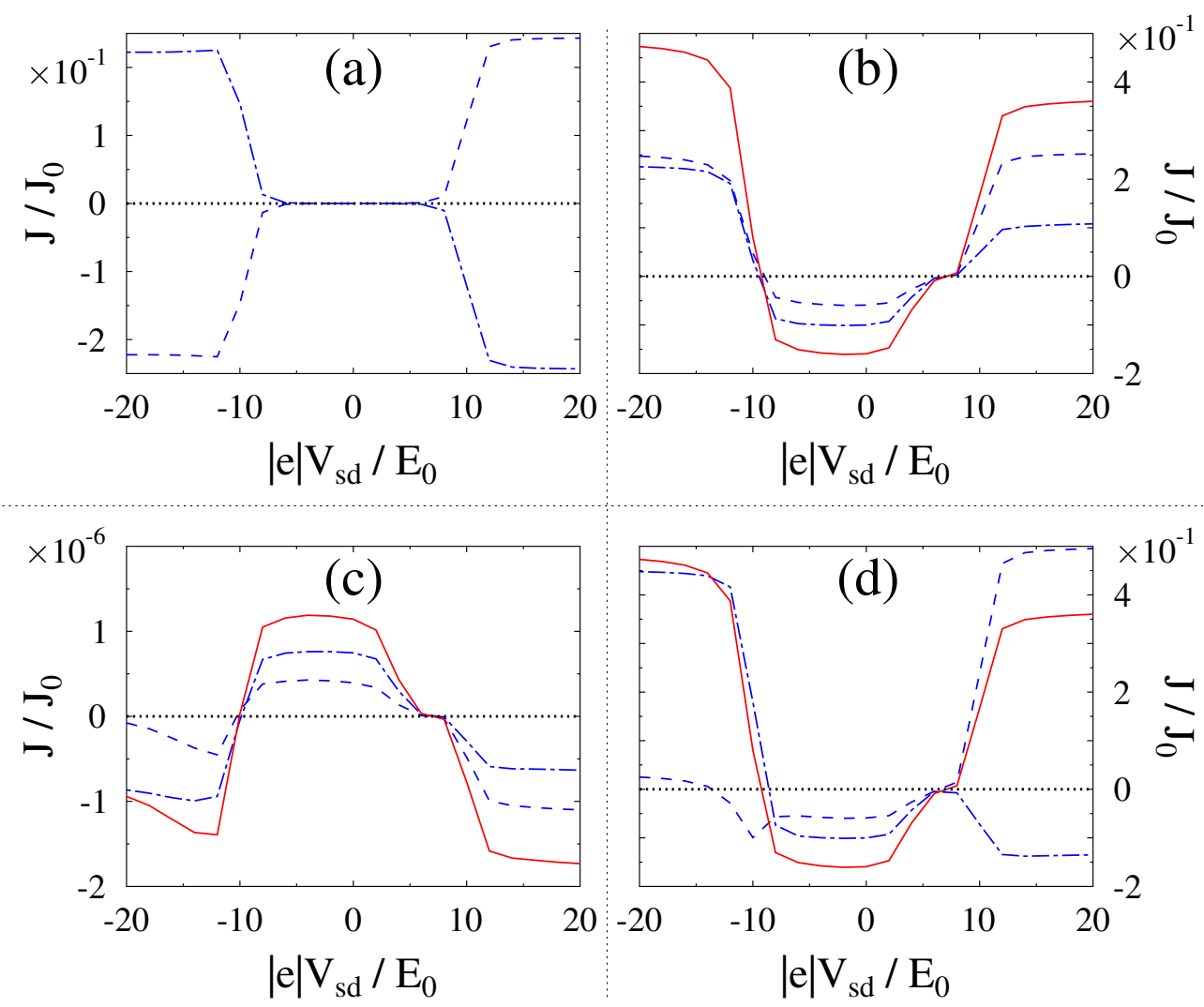

Figure 7: Energy conservation, eq 35, for the junction model of Figure 5 . Shown are $J_{L}$ (dashed line, blue), $J_{R}$ (dash-dotted line, blue), $J_{p t}$ (solid line, red) and their sum (dotted line, black) for (a) zero, (b) second. and (c) fourth order contributions; (d) shows total fluxes, eqs 38 and 39. See text for parameters. 
levels populations difference at consecutive steps is less than $10^{-12}$. Results for particle and energy fluxes are presented in terms of flux units $I_{0} \equiv 1 / t_{0}$ and $J_{0} \equiv E_{0} / t_{0}$, respectively $\left(t_{0} \equiv \hbar / E_{0}\right.$ is unit of time).
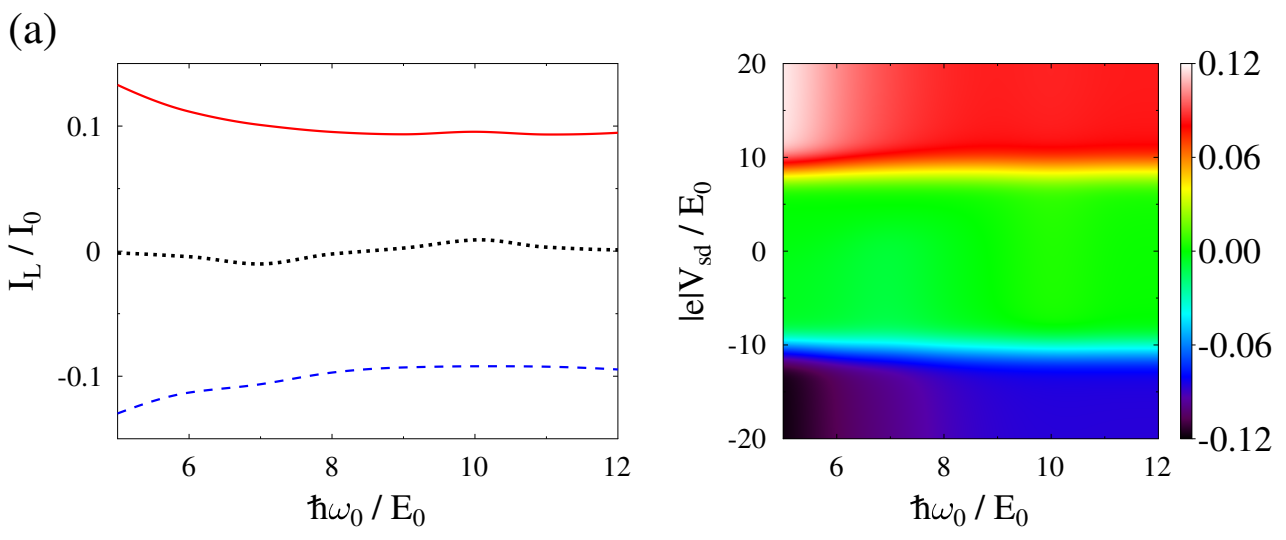

(b)
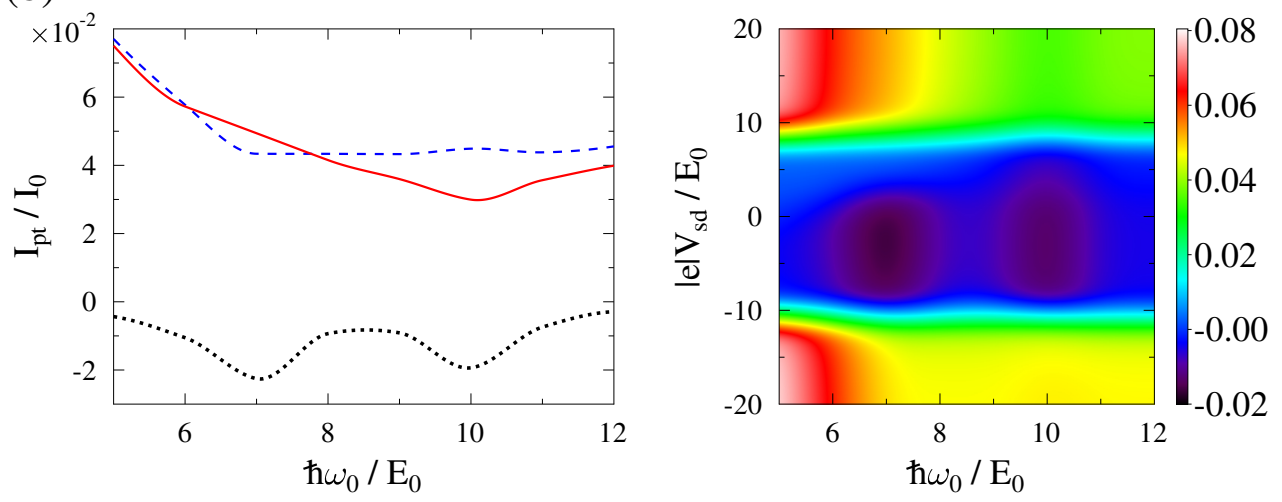

Figure 8: Particle fluxes vs. pumping frequency $\omega_{0}$ for the junction model of Figure 5 . Shown are (a) $I_{L}=-I_{R}$, eq 36, and (b) $I_{p t}$, eq 37, at biases $|e| V_{s d}=-16 E_{0}$ (dashed line, blue), $|e| V_{s d}=0$ (dotted line, black), and $|e| V_{s d}=16 E_{0}$ (solid line, red) in left panels. Right column shows map of the fluxes vs. pumping frequency $\omega_{0}$ and bias $V_{s d}$. See text for parameters.

Figure 6 shows charge currents, eq 36, at the left and right interfaces (dashed and dash=dotted lines, respectively). Their sum (dotted line) by charge conservation, eq 34 . should be zero at steady-state. Panels (a)-(c) present contributions to the fluxes of the zero, second, and fourth order diagrams in molecule-radiation field coupling strength; panel (d) shows sum of all the contributions. Similarly, Figure 7 shows energy currents due to electrons, eq 38 at the left (dashed line) and the right (dash-dotted line) interfaces and due to photons (solid line), eq 39. Their sum (dotted line) by energy conservation, eq 35 , is zero at 

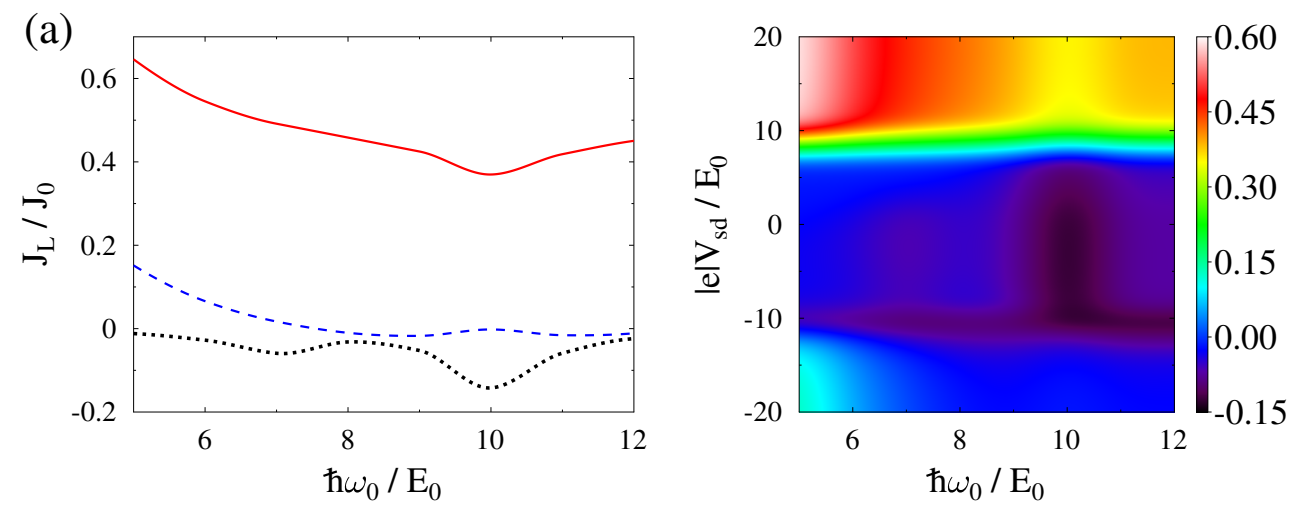

(b)
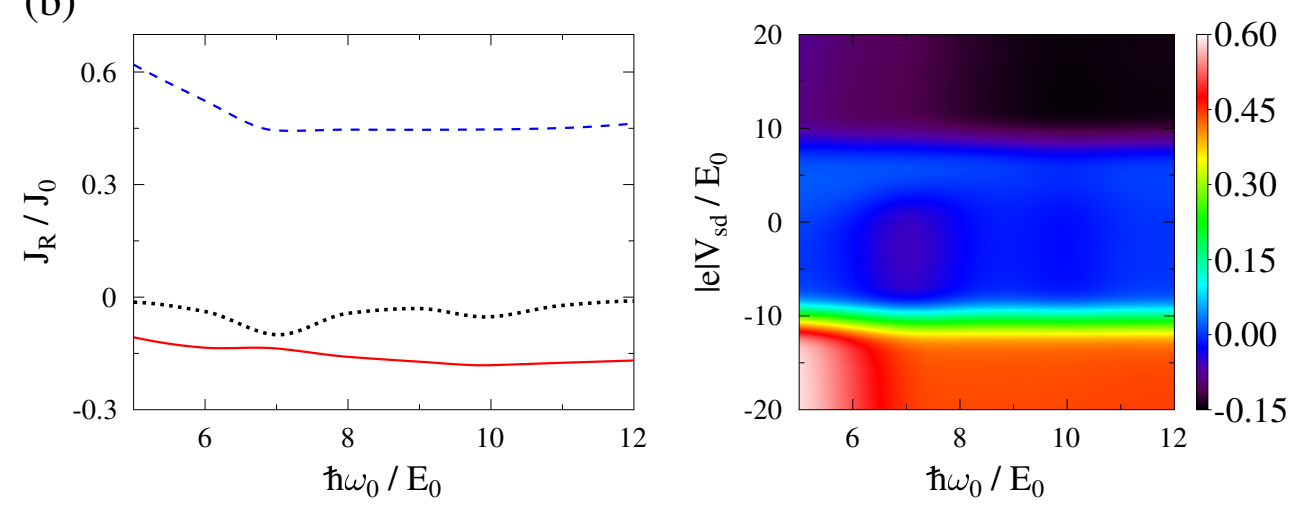

(c)
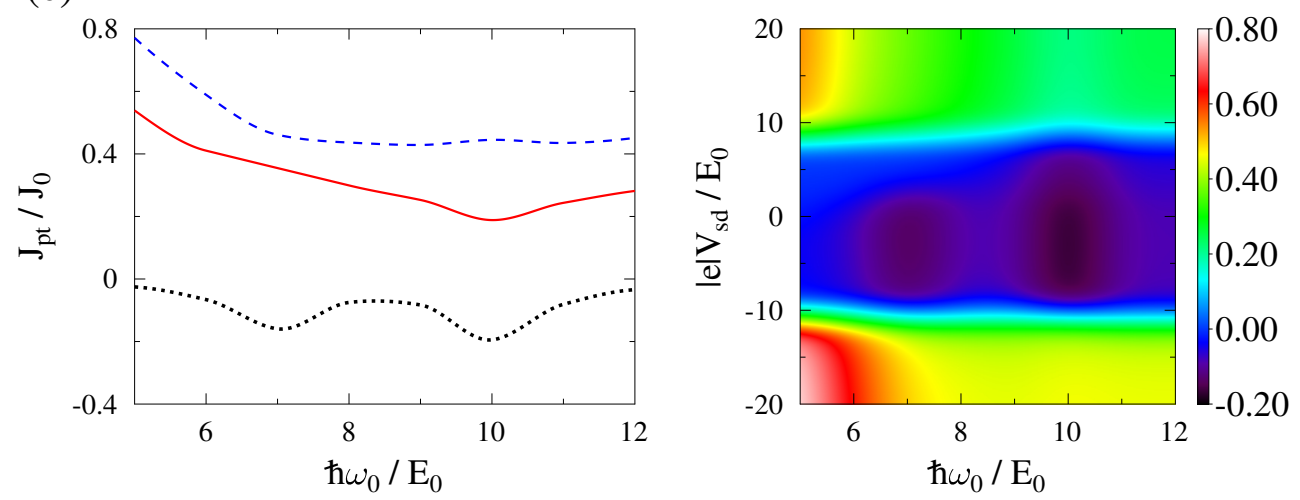

Figure 9: Energy fluxes vs. pumping frequency $\omega_{0}$ for the junction model of Figure 5 . Shown are (a) $J_{L}$, eq 38, (b) $J_{R}$, eq 38, and (c) $J_{p t}$, eq 39, at biases $|e| V_{s d}=-16 E_{0}$ (dashed line, blue), $|e| V_{s d}=0$ (dotted line, black), and $|e| V_{s d}=16 E_{0}$ (solid line, red) in left panels. Right column shows map of the fluxes vs. pumping frequency $\omega_{0}$ and bias $V_{s d}$. See text for parameters. 
steady-state. Note that the conservation laws are satisfied at each order of our diagrammatic expansion in light matter interaction, i.e. the sum of all double sided Feynman diagrams of a particular order satisfies charge and energy conservation.

Figures 8 and 9 present spectroscopy of particle, eqs 36 and 37, and energy, eqs 38 and 39. fluxes for the junction model of Figure 5. While for the choice of parameters charge current (Figure 8a) mostly depends on bias, the photon flux (Figure 8b) is sensitive to the radiation field frequency. At zero bias the photon flux has dips at molecular resonances $\varepsilon_{2}-\varepsilon_{1}=10 E_{0}$ and $\varepsilon_{2}-\varepsilon_{3}=7 E_{0}$ due to photon absorption by the electronic system (see dotted line and map in Fig. 8p). At higher biases laser induced absorption competes with bias induced emission. Thus, the photon flux is suppressed at molecular resonances (see solid an dashed line in Fig. 8b).

The energy fluxes show a similar frequency dependence. In particular, dips in $J_{L}, J_{R}$ and $J_{p t}$ at donor-bridge molecular resonance $\varepsilon_{2}-\varepsilon_{1}=10$ and $\varepsilon_{2}-\varepsilon_{1}=10$ at low biases (see dotted lines and maps in Figures $9 \mathrm{a}, \mathrm{b}$ and c) indicate increased $L$ to $M$ and $R$ to $M$ energy fluxes caused by increased electron transfer into the donor and acceptor facilitated by the radiation field pumping. Note that for $\varepsilon_{1}=-5 E_{0}$ and $\varepsilon_{3}=-2 E_{0}$ energy flux coming from the contacts will be negative. Similarly, dips in the photon flux indicate increase in energy coming into the system. At higher biases radiation field pumping is counteracted by bias induced emission, so that the energy curves become smoother, although $J_{L}$ and $J_{p t}$ still show dips at molecular resonance corresponding to donor-bridge transition (see solid lines in Figures $9 \mathrm{a}$ and $\mathrm{c}$ ).

Note that while in the numerical illustration we focus on steady-state, where the initial state of the field and way of switching on of the light-matter interaction are not important, description of light pulses will require solving corresponding time-dependent problem, eqs 25 . 28 


\section{Conclusions}

We had developed a theoretical description of optical spectroscopy for open nonequilibrium systems, where both molecular degrees of freedom and radiation field are treated quantum mechanically and where charge and energy conservations in the system are built in. Starting from nonequilibrium Green's function formulations we show connection with Liouville space description and introduce generalization of double-sided Feynman diagrams. The latter is standard tool widely used by theorists and experimentalists for design and interpretation of experiments.

We performed an expansion in the light-matter coupling strength within the standard NEGF, and presented different contributions to the photon flux by double-sided Feynman diagrams. In particular, the order of diagrammatic expansion in photon self-energy due to coupling to electrons is identified as order of optical process. Double-sided Feynman diagrams are shown to be projections of corresponding Feynman diagrams on the Keldysh contour. Light-matter interaction events in double-sided Feynman diagrams are accompanied by change of molecular orbital, as is expected for weak coupling case.

Our study bridges the theoretical approaches used in quantum transport and optical spectroscopy. It establishes firm theoretical basis for applying traditional tools of nonlinear optical spectroscopy in molecular optoelectronics. Developing theoretical description of optical spectroscopy for strongly interacting open molecular systems is a goal for future research.

\section{Supporting Information Available}

The following files are available free of charge. Supporting information. Explicit expressions for the self-energies of electrons due to coupling to radiation field modes and photons due to coupling to electrons to fourth order in light-matter interaction. 


\section{Acknowledgement}

This material is based upon work supported by the National Science Foundation under Grants No. CHE-1565939 (M.G.) and No. CHE-1663822 (S.M.).

\section{References}

(1) Krausz, F.; Ivanov, M. Attosecond Physics. Rev. Mod. Phys. 2009, 81, 163-234.

(2) Kawata, S.; Inouye, Y.; Verma, P. Plasmonics for Near-Field Nano-Imaging and Superlensing. Nature Photon. 2009, 3, 388-394.

(3) Le Ru, E. C.; Etchegoin, P. G. Single-Molecule Surface-Enhanced Raman Spectroscopy. Ann. Rev. Phys. Chem. 2012, 63, 65-87.

(4) Verma, P. Tip-Enhanced Raman Spectroscopy: Technique and Recent Advances. Chem. Rev. 2017, 117, 6447-6466.

(5) Kampfrath, T.; Tanaka, K.; Nelson, K. A. Resonant and Nonresonant Control Over Matter and Light by Intense Terahertz Transients. Nature Photon. 2013, 7, 680-690.

(6) Young, L.; Ueda, K.; Gühr, M.; Bucksbaum, P. H.; Simon, M.; Mukamel, S.; Rohringer, N.; Prince, K. C.; Masciovecchio, C.; Meyer, M. et al. Roadmap of Ultrafast X-ray Atomic and Molecular Physics. J. Phys. B: At., Mol. Opt. Phys. 2018, $51,032003$.

(7) Bennett, K.; Kowalewski, M.; Rouxel, J. R.; Mukamel, S. Monitoring Molecular Nonadiabatic Dynamics with Femtosecond X-ray Diffraction. Proc. Natl. Acad. Sci. 2018, $115,6538-6547$.

(8) Schlawin, F.; Dorfman, K. E.; Mukamel, S. Entangled Two-Photon Absorption Spectroscopy. Acc. Chem. Res. 2018, 51, 2207-2214. 
(9) Dorfman, K. E.; Asban, S.; Ye, L.; Rouxel, J. R.; Cho, D.; Mukamel, S. Monitoring Spontaneous Charge-Density Fluctuations by Single-Molecule Diffraction of Quantum Light. J. Phys. Chem. Lett. 2019, 10, 768-773.

(10) Asban, S.; Dorfman, K. E.; Mukamel, S. Quantum Phase-Sensitive Diffraction and Imaging Using Entangled Photons. Proc. Natl. Acad. Sci. 2019, 201904839.

(11) Qiu, X. H.; Nazin, G. V.; Ho, W. Vibrationally Resolved Fluorescence Excited with Submolecular Precision. Science 2003, 299, 542-546.

(12) Dong, Z.-C.; Guo, X.-L.; Trifonov, A. S.; Dorozhkin, P. S.; Miki, K.; Kimura, K.; Yokoyama, S.; Mashiko, S. Vibrationally Resolved Fluorescence from Organic Molecules near Metal Surfaces in a Scanning Tunneling Microscope. Phys. Rev. Lett. 2004, 92, 086801.

(13) Wu, S. W.; Nazin, G. V.; Ho, W. Intramolecular Photon Emission from a Single Molecule in a Scanning Tunneling Microscope. Phys. Rev. B 2008, 77, 205430.

(14) Chen, C.; Chu, P.; Bobisch, C. A.; Mills, D. L.; Ho, W. Viewing the Interior of a Single Molecule: Vibronically Resolved Photon Imaging at Submolecular Resolution. Phys. Rev. Lett. 2010, 105, 217402.

(15) Zhang, Y.; Luo, Y.; Zhang, Y.; Yu, Y.-J.; Kuang, Y.-M.; Zhang, L.; Meng, Q.-S.; Luo, Y.; Yang, J.-L.; Dong, Z.-C. et al. Visualizing Coherent Intermolecular DipoleDipole Coupling in Real Space. Nature 2016, 531, 623-627.

(16) Imada, H.; Miwa, K.; Imai-Imada, M.; Kawahara, S.; Kimura, K.; Kim, Y. Real-Space Investigation of Energy Transfer in Heterogeneous Molecular Dimers. Nature 2016, 538, 364-367.

(17) Kimura, K.; Miwa, K.; Imada, H.; Imai-Imada, M.; Kawahara, S.; Takeya, J.; 
Kawai, M.; Galperin, M.; Kim, Y. Selective Triplet Exciton Formation in a Single Molecule. Nature 2019, 570, 210-213.

(18) Schneider, N. L.; Lü, J. T.; Brandbyge, M.; Berndt, R. Light Emission Probing Quantum Shot Noise and Charge Fluctuations at a Biased Molecular Junction. Phys. Rev. Lett. 2012, 109, 186601.

(19) Natelson, D.; Li, Y.; Herzog, J. B. Nanogap Structures: Combining Enhanced Raman Spectroscopy and Electronic Transport. Phys. Chem. Chem. Phys. 2013, 15, 52625275 .

(20) Ward, D. R.; Halas, N. J.; Ciszek, J. W.; Tour, J. M.; Wu, Y.; Nordlander, P.; Natelson, D. Simultaneous Measurements of Electronic Conduction and Raman Response in Molecular Junctions. Nano Lett. 2008, 8, 919-924.

(21) Ioffe, Z.; Shamai, T.; Ophir, A.; Noy, G.; Yutsis, I.; Kfir, K.; Cheshnovsky, O.; Selzer, Y. Detection of Heating in Current-Carrying Molecular Junctions by Raman Scattering. Nature Nanotech. 2008, 3, 727-732.

(22) Ward, D. R.; Corley, D. A.; Tour, J. M.; Natelson, D. Vibrational and Electronic Heating in Nanoscale Junctions. Nature Nanotech. 2011, 6, 33-38.

(23) Grosse, C.; Etzkorn, M.; Kuhnke, K.; Loth, S.; Kern, K. Quantitative Mapping of Fast Voltage Pulses in Tunnel Junctions by Plasmonic Luminescence. Appl. Phys. Lett. 2013, 103, 183108.

(24) Galperin, M.; Nitzan, A. Molecular Optoelectronics: The Interaction of Molecular Conduction Junctions with Light. Phys. Chem. Chem. Phys. 2012, 14, 9421-9438.

(25) Okumura, K.; Tanimura, Y. The $(2 \mathrm{n}+1)$ th-order Off-Resonant Spectroscopy from the $(\mathrm{n}+1)$ th-order Anharmonicities of Molecular Vibrational Modes in the Condensed Phase. J. Chem. Phys. 1997, 106, 1687-1698. 
(26) Okumura, K.; Tanimura, Y. Femtosecond Two-Dimensional Spectroscopy from Anharmonic Vibrational Modes of Molecules in the Condensed Phase. J. Chem. Phys. 1997, 107, 2267-2283.

(27) Xu, Q.-H.; ; Fleming, G. R. Isomerization Dynamics of 1,1-Diethyl-4,4-Cyanine (1144C) Studied by Different Third-Order Nonlinear Spectroscopic Measurements. J. Phys. Chem. A 2001, 105, 10187-10195.

(28) Ovchinnikov, M.; Apkarian, V. A.; Voth, G. A. Semiclassical Molecular Dynamics Computation of Spontaneous Light Emission in the Condensed Phase: Resonance Raman Spectra. J. Chem. Phys. 2001, 114, 7130-7143.

(29) Okumura, K.; Tanimura, Y. Energy-Level Diagrams and Their Contribution to FifthOrder Raman and Second-Order Infrared Responses: Distinction between Relaxation Models by Two-Dimensional Spectroscopy. J. Phys. Chem. A 2003, 107, 8092-8105.

(30) Mukamel, S.; ; Abramavicius, D. Many-Body Approaches for Simulating Coherent Nonlinear Spectroscopies of Electronic and Vibrational Excitons. Chem. Rev. 2004, 104, 2073-2098.

(31) Šanda, F.; Mukamel, S. Liouville-Space Pathways for Spectral Diffusion in Photon Statistics from Single Molecules. Phys. Rev. A 2005, 71, 033807.

(32) Yang, L.; Mukamel, S. Revealing Exciton-Exciton Couplings in Semiconductors Using Multidimensional Four-Wave Mixing Signals. Phys. Rev. B 2008, 77, 075335.

(33) Harbola, U.; Mukamel, S. Coherent Stimulated X-ray Raman Spectroscopy: Attosecond Extension of Resonant Inelastic X-ray Raman Scattering. Phys. Rev. B 2009, 79, 085108.

(34) Mukamel, S. Principles of Nonlinear Optical Spectroscopy; Oxford University Press, 1995. 
(35) Breuer, H.-P.; Petruccione, F. The Theory of Open Quantum Systems; Oxford University Press, 2003.

(36) Gao, Y.; Galperin, M. Simulation of Optical Response Functions in Molecular Junctions. J. Chem. Phys. 2016, 144, 244106.

(37) Galperin, M. Photonics and Spectroscopy in Nanojunctions: A Theoretical Insight. Chem. Soc. Rev. 2017, 46, 4000-4019.

(38) Roslyak, O.; Mukamel, S. Lectures of Virtual University, Max Born Institute, EVU Lecture Notes.

(39) Baym, G.; Kadanoff, L. P. Conservation Laws and Correlation Functions. Phys. Rev. 1961, 124, 287-299.

(40) Baym, G. Self-Consistent Approximations in Many-Body Systems. Phys. Rev. 1962, 127, 1391-1401.

(41) Kadanoff, L. P.; Baym, G. In Quantum Statistical Mechanics; Pines, D., Ed.; Frontiers in Physics; W. A. Benjamin, Inc.: New York, 1962.

(42) Gao, Y.; Galperin, M. Optical Spectroscopy of Molecular Junctions: Nonequilibrium Green's Functions Perspective. J. Chem. Phys. 2016, 144, 174113.

(43) Nitzan, A.; Galperin, M. Kinetic Schemes in Open Interacting Systems. J. Phys. Chem. Lett. 2018, 9, 4886-4892.

(44) Haug, H.; Jauho, A.-P. Quantum Kinetics in Transport and Optics of Semiconductors; Springer: Berlin Heidelberg, 2008.

(45) Stefanucci, G.; van Leeuwen, R. Nonequilibrium Many-Body Theory of Quantum Systems. A Modern Introduction.; Cambridge University Press, 2013. 
(46) Luttinger, J. M.; Ward, J. C. Ground-State Energy of a Many-Fermion System. II. Phys. Rev. 1960, 118, 1417-1427.

(47) Haussmann, R. Self-consistent Quantum-Field Theory and Bosonization for Strongly Correlated Electron Systems; Springer-Verlag: Berlin Heidelberg, 1999.

(48) Stan, A.; Dahlen, N. E.; van Leeuwen, R. Time propagation of the KadanoffBaym equations for inhomogeneous systems. J. Chem. Phys. 2009, 130, 224101.

(49) Latini, S.; Perfetto, E.; Uimonen, A.-M.; van Leeuwen, R.; Stefanucci, G. Charge dynamics in molecular junctions: Nonequilibrium Green's function approach made fast. Phys. Rev. B 2014, 89, 075306.

(50) Jauho, A.-P.; Wingreen, N. S.; Meir, Y. Time-Dependent Transport in Interacting and Noninteracting Resonant-Tunneling Systems. Physical Review B 1994, 50, 5528-5544.

(51) Galperin, M.; Nitzan, A.; Ratner, M. A. Heat Conduction in Molecular Transport Junctions. Phys. Rev. B 2007, 75, 155312.

(52) Bergmann, N.; Galperin, M. Electron Transfer Methods in Open Systems. J. Phys. Chem. B 2019, 123, 7225-7232.

(53) Leijnse, M.; Wegewijs, M. R. Kinetic Equations for Transport Through Single-Molecule Transistors. Phys. Rev. B 2008, 78, 235424.

(54) Koller, S.; Grifoni, M.; Leijnse, M.; Wegewijs, M. R. Density-Operator Approaches to Transport Through Interacting Quantum Dots: Simplifications in Fourth-Order Perturbation Theory. Phys. Rev. B 2010, 82, 235307. 


\section{Graphical TOC Entry}

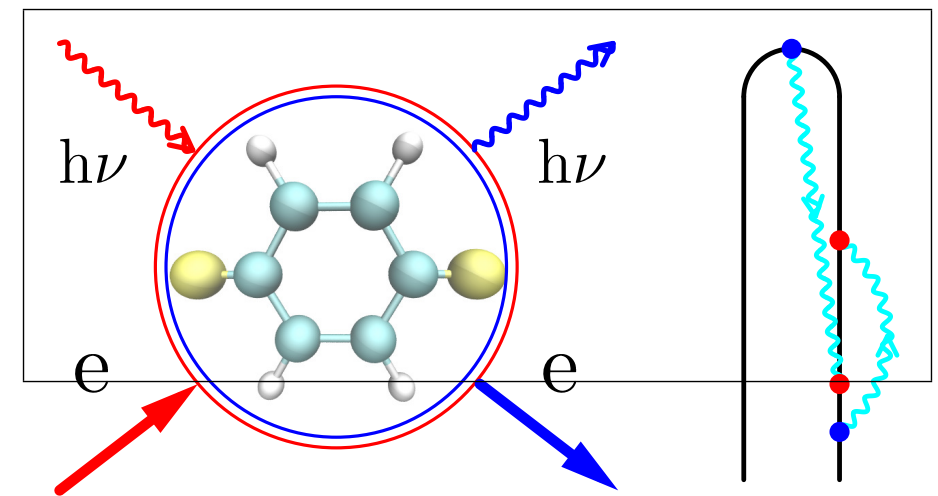

Conserving diagrammatic formulation of optical spectroscopy of open quantum systems. 